\title{
Fourth Ventricular Outflow Obstruction in an Infant with Ileal Atresia and Laryngomalacia: Endoscopic Management
}

\author{
Forhad H. Chowdhury ${ }^{1}$ Mohammod Raziul Haque ${ }^{2}$ \\ Mohammod Samsul Arifin ${ }^{3}$ \\ ${ }^{1}$ Department of Neurosurgery, National Institute of Neurosciences \\ and Hospital, Dhaka, Bangladesh \\ 2Department of Neurosurgery, Dhaka Medical College Hospital, \\ Dhaka, Bangladesh \\ ${ }^{3}$ Department of Neurosurgery, Ibn Sina Specialized Hospital, \\ Dhanmondi, Dhaka, Bangladesh
}

Jalal Uddin Mohammod Rumi ${ }^{1}$

Indian J Neurosurg:2020;9:219-224

\begin{abstract}
Address for correspondence Forhad H. Chowdhury, MBBS, FCPS, MS, Department of Neurosurgery, National Institute of Neurosciences and Hospital, Shere-e-Bangla Nagar, Dhaka 1207, Bangladesh (e-mail: forhadchowdhury74@yahoo.com).
\end{abstract}

\begin{abstract}
Keywords

- hydrocephalus

- fourth ventricle outflow obstruction

- endoscopic third ventriculostomy

- re-endoscopic procedure

Fourth ventricle outflow obstruction (FVOO) is a rare cause of obstructive hydrocephalus. In this study, we described a case of idiopathic FVOO with ileal atresia and laryngomalacia which was managed with endoscopic third ventriculostomy (ETV) and re-endoscopy. We also described the techniques of fenestration of Liliequist membrane and partial removal of arachnoid membrane over dorsum sella (DS) to prevent closure of fenestration and recurrence of hydrocephalus. The patient was a 4-month-old infant presented with progressively increasing head size, feeding difficulty, respiratory distress, and tense fontanel. The infant had a history of laparotomy for ileal atresia. CT scan showed panventriculomegaly due to FVOO. ETV with fenestration of Liliequist membrane was done on emergency basis. After operation, the patient improved clinically and radiologically. Four weeks later, the patient returned with recurrent hydrocephalus. Endoscopic reoperation showed closure of fenestration in arachnoid membrane (Lilieqiest membrane). Endoscopic refenestration with partial excision of arachnoid on DS was done. The patient again recovered radiologically and clinically till last follow-up. In idiopathic FVOO, ETV with wide fenestration of Liliequist membrane, preferably with partial removal of arachnoid on DS, may be very useful in treating hydrocephalus (HCP) and preventing recurrent $\mathrm{HCP}$ even in infants.
\end{abstract}

\section{Introduction}

Fourth ventricle outflow obstruction (FVOO) is a rare clinico-radiological condition, which causes obstructive hydrocephalus. In FVOO, cerebrospinal fluid (CSF) flow is blocked at the foramen Magendie and Luschka due to atresia or by a membrane in the absence of any other obstruction of CSF flow. Various terms for FVOO have been used such as fourth ventricle/ventricular outlet obstruction, ${ }^{1-3}$ fourth ventricular outflow obstruction, ${ }^{4}$ membranous obstruction of the fourth ventricle outlet, ${ }^{5}$ obstruction of Magendie's and Luschka's foramina, ${ }^{6}$ obstruction of fourth ventricular exit, ${ }^{7}$ and primary obstruction of the fourth ventricle outlets. ${ }^{8}$ Far distal obstructive HCP is a term that includes Dandy Walker or Arnold Chiari malformation, membranous obstruction of fourth ventricle, and intercisternal external obstruction of the CSF. ${ }^{9}$ The etiopathogenesis of FVOO is not well understood and may be congenital, although some cases present with a history of meningitis or intraventricular hemorrhage.
DOI https://doi.org/ $10.1055 / \mathrm{s}-0040-1710104$ ISSN 2277-954X.

\section{(C) 2020. Neurological Surgeons' Society of India.}

This is an open access article published by Thieme under the terms of the Creative Commons Attribution-NonDerivative-NonCommercial-License, permitting copying and reproduction so long as the original work is given appropriate credit. Contents may not be used for commercial purposes, or adapted, remixed, transformed or built upon. (https://creativecommons.org/licenses/by-nc-nd/4.0/)

Thieme Medical and Scientific Publishers Pvt. Ltd., A-12, 2nd Floor, Sector 2, Noida-201301 UP, India 
Here, we described a technical case of neonatal idiopathic FVOO with ileal atresia and laryngomalacia where initial endoscopic third ventriculostomy (ETV) was successful but re-endoscopic procedure was needed for recurrence of HCP.

\section{Case Presentation}

Our patient was 4 months of age, first issue of his parents (nonconsanguineus marriage) presented with progressively increasing head size, feeding difficulty, respiratory distress, and tense fontanel. The infant had a history of $(\mathrm{H} / \mathrm{O})$ laparotomy in the 1st week of his life for ileal atresia where ileal resection and ileo-ileal anastomosis with proximal ileostomy were done. Ileostomy was closed when he reached his 8th week. He was also diagnosed with a case of laryngomalacia. He had no history of birth asphyxia, head trauma, or central nervous system (CNS) infection.

The patient came to us with a previously done (10 days earlier) CT scan of head, which showed dilatation of all ventricles with ballooning of fourth ventricle, and foramen of Magendie and Luschka. The cerebellum was hypoplastic and brainstem was pushed anteriorly. There was no visible cisterna magna, premedullary cistern, prepontine cistern, or basal cistern. Brain parenchyma was pushed toward the periphery and there was periventricular hypodensity (-Fig. 1). So, a diagnosis of FVOO was made. A repeat CT scan was done immediately which showed all the earlier-mentioned findings in augmented form (-Fig. 2). The previously done echocardiogram and ultrasonography (USG) of whole abdomen were normal. After counseling, emergency ETV was done under G/A.

\section{Operation ETV}

Under general anesthesia with endotracheal intubation, patient was positioned in supine position. Through the precoronal rightlateral part of the anterior fontanel, the lateral ventricle was entered. Through the right foramen of Monro, the third ventricle

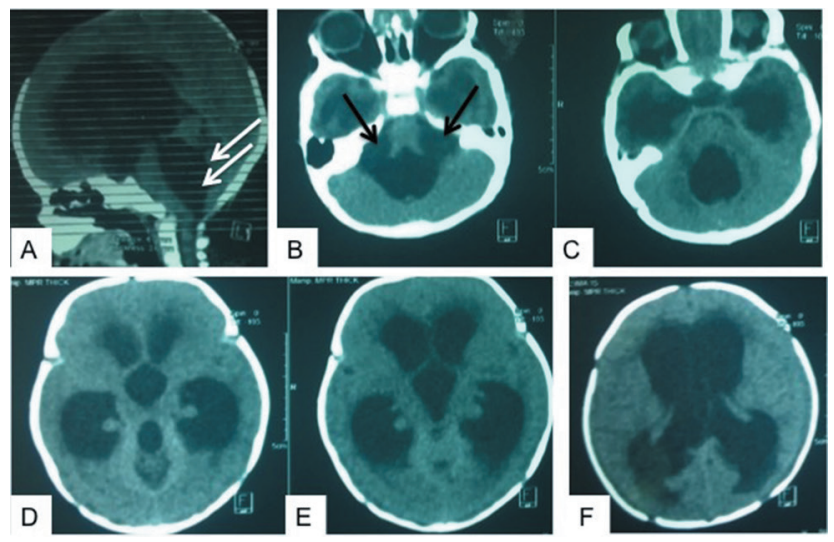

Fig. 1 CT scan of head that was done 10 days earlier to presentation to us: (A) sagittal section, (B-F) axial sections showing panventriculomegaly due to FVOO with periventricular edema. Arrow marks in (A) showing fourth ventricular enlargement with absent of cisterna magna, indicating foramen Magendie atresia. Arrow marks in (B) showing enlargement of lateral recesses of fourth ventricle as well as absent cerebellopontine angle cisterns, indicating foramen Luschka atresia. FVOO, fourth ventricular outflow obstruction. was reached. After identification of mammillary bodies, infundibular recess, basilar bifurcation, and dorsum sella ([DS] through the ventricular floor), a fenestration was made very carefully with endoscopic ventricular forceps in the midline floor of the third ventricle between DS and basilar artery (BA). Then, the space between DS and BA was increased due to CSF entry from ventricle and that made the rest of the operation more feasible. The fenestration was enlarged with 3F Fogarthy catheter balloon. The thick layer of arachnoid band was found attached to the DS to BA, which was also widely fenestrated and partially excised (-Fig. 3). Clean pulsating CSF flow was seen through the fenestration. Endoscope was removed after inspection of

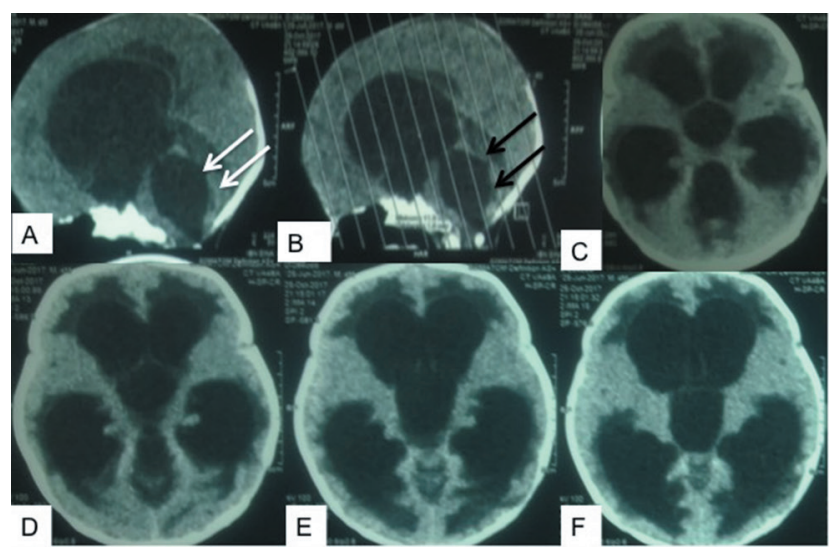

Fig. 2 CT scan of head at presentation: (A, B) sagittal sections and (C-F) axial sections, showing augmented form of panventriculomegaly due to FVOO with increased periventricular edema. Arrow marks in (A, B) showing fourth ventricular enlargement with absence of cisterna magna, indicating foramen Magendie atresia. FVOO, fourth ventricular outflow obstruction.

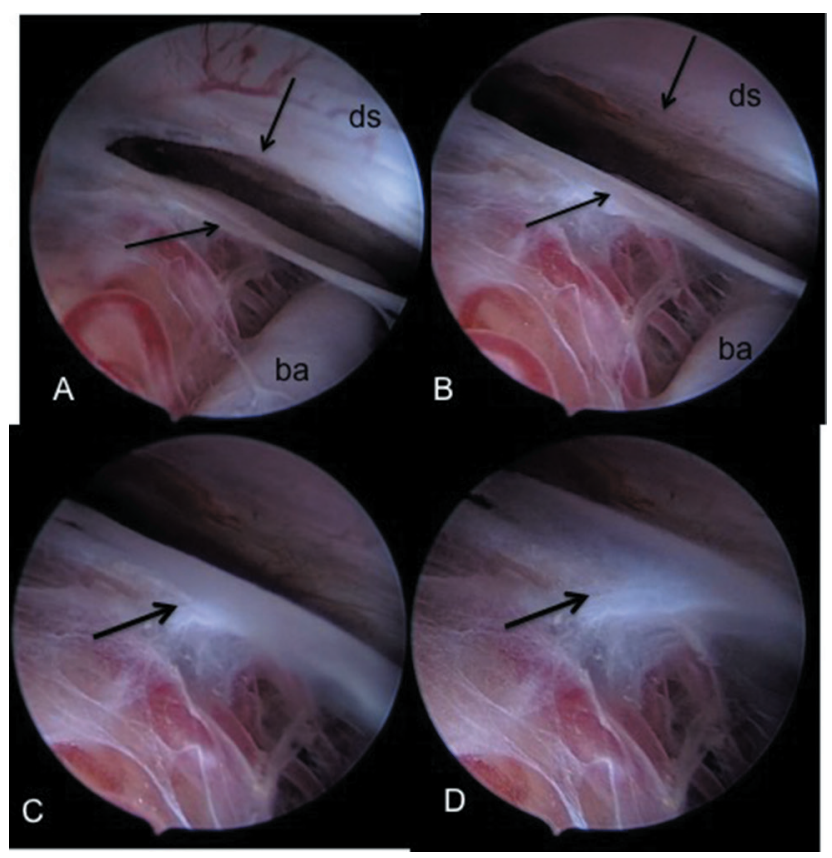

Fig. 3 Peroperative pictures of 1st operation ETV: thick floating arachnoid layer anterior to BA and posterior to clivus and DS, as indicated by arrow marks (which reunited [seen during 2nd endoscopy] and hydrocephalus recurred). BA, basilar artery; DS, dorsum sella; ETV, endoscopic third ventriculostomy. 

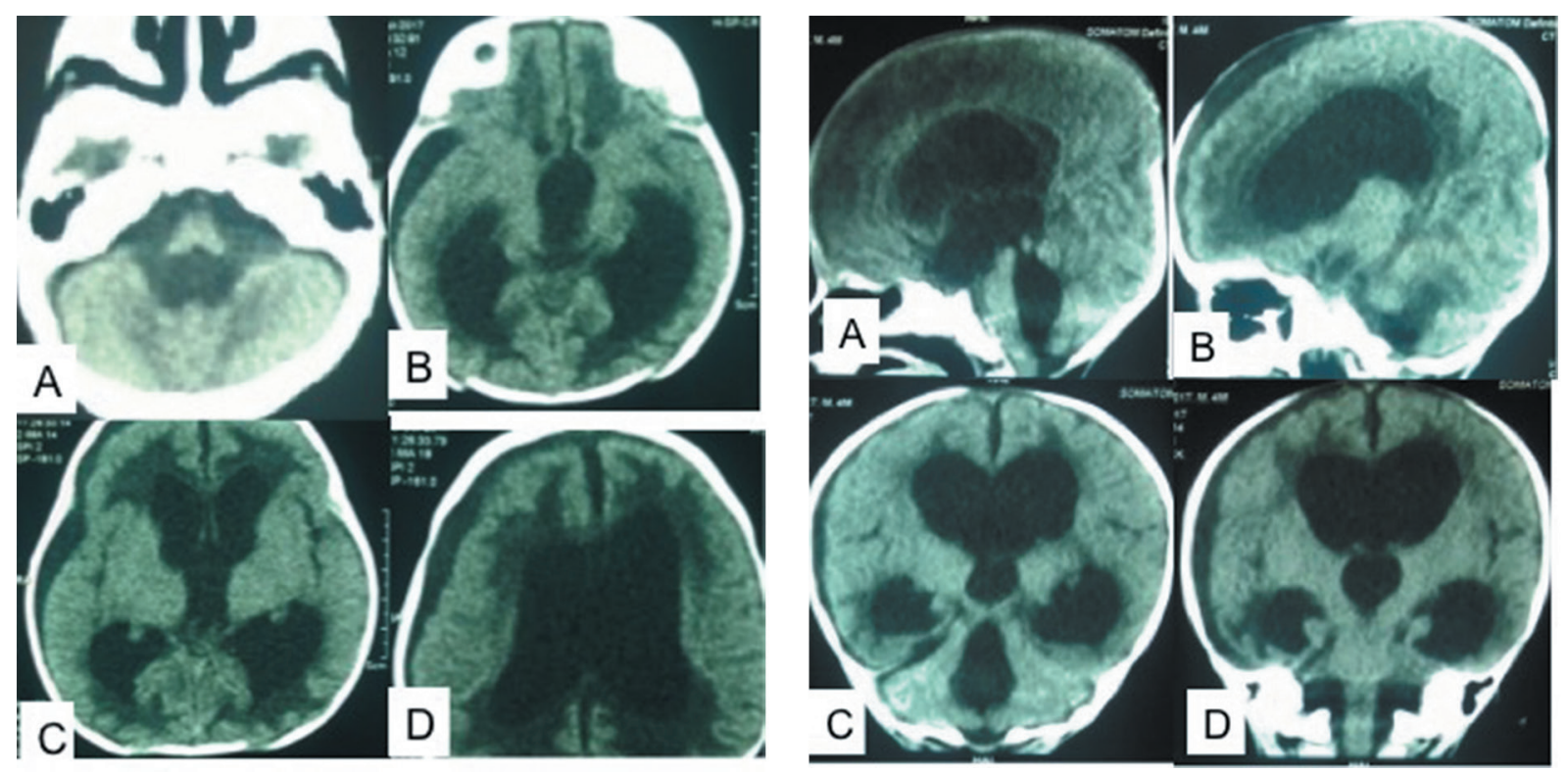

Fig. 4 (A) CT scan of head axial sections (A,B,C, and D) was done on 1st postoperative day after ETV, showing decreased ventricular size with opening of subarachnoid spaces, especially on right side. (B) CT scan of head: (A, B) sagittal sections and (C, D) coronal sections that was done on 1st postoperative day after ETV, showing decreased ventricular size with opening of subarachnoid spaces, especially on right side.

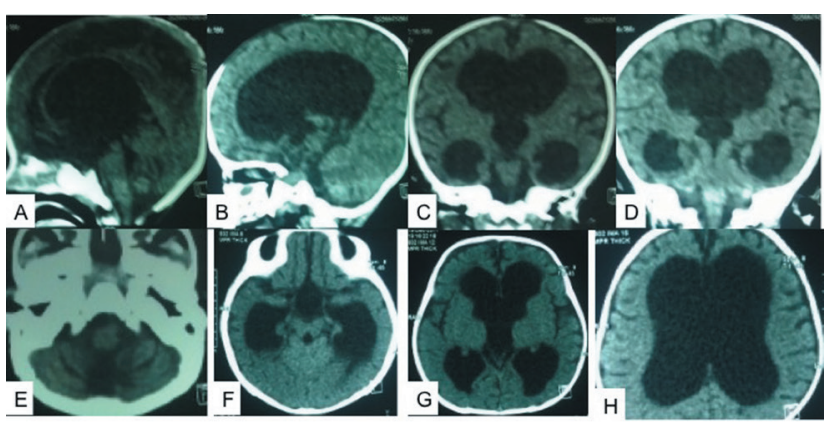

Fig. 5 CT scan of head that was done 4 weeks after ETV: (A, B) sagittal sections, (C, D) coronal sections, and (E-H) axial sections showing recurrence of panventriculomegaly with periventricular edema as well as absence of subarachnoid spaces. ETV, endoscopic third ventriculostomy.

cerebral aqueduct (which was open and enlarged) and wound closed accordingly.

Postoperatively, the patient became stable with respiration and breast feeding. His fontanel became lax. CT scan of head on 1st postoperative day showed decrease size of ventricles, including fourth ventricle with CSF in subarachnoid spaces which was more marked on the right side ( - Fig. 4 A, B).

After 4 weeks of ETV, the patient again developed feeding and respiratory difficulties with tense anterior fontanel. Repeat CT scan showed ventricular re-enlargement including fourth ventricle with periventricular hypodensity; brain parenchyma was pushed toward periphery with drained subarachnoid spaces ( $\mathbf{- F i g}$. 5). We decided to reoperate on him after counseling with parents regarding the necessity of shunt operation if re-endoscopy fails.

\section{Re-endoscopic Operation}

Under general anesthesia with endotracheal intubation patient in supine position, ventricle was reached through

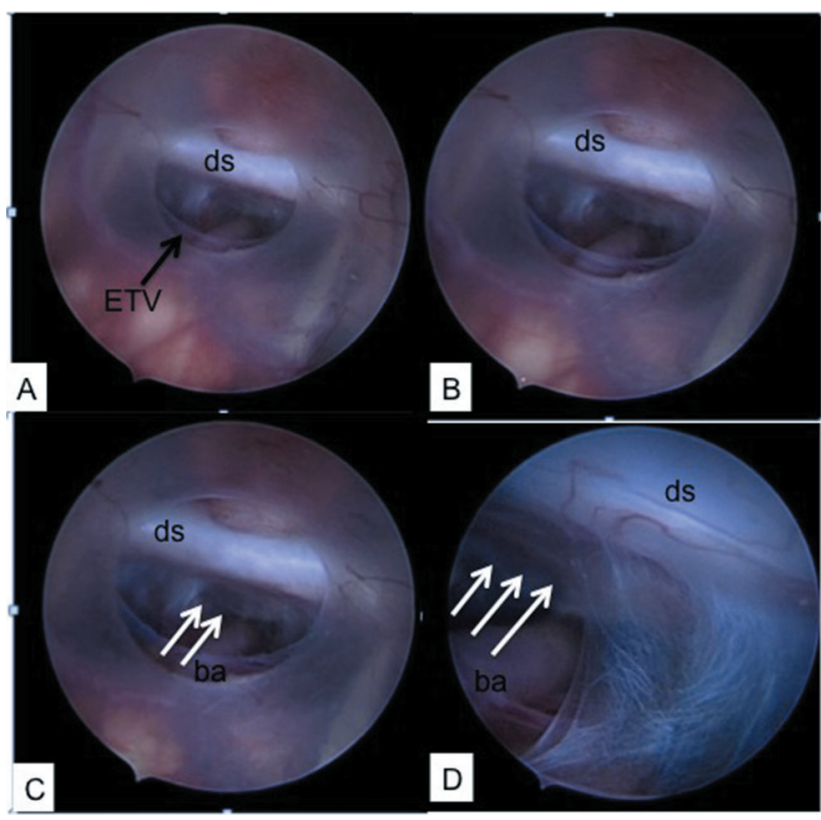

Fig. 6 (A-D) Peroperative pictures showing ETV stoma was patent but arachnoid fenestration between BA and DS was obliterated to stop CSF flow from the third ventricle. Arrow marks showing arachnoid reunion site (thick arachnoid flap seen during first operation between BA and DS reunited with arachnoid over DS). BA, basilar artery; DS, dorsum sella; CSF, cerebrospinal fluid; ETV, endoscopic third ventriculostomy.

the previous route. Endoscopic stoma in the floor of the third ventricle was patent but there was no flow through the stoma. Arachnoid membranes were uniformly reunited to a membrane between DS and brainstem (including BA; - Fig. 6). With the boring action of the tip of the Fogarthy catheter, we perforated the membrane between clivus and BA. With the help of endoscopic grasping forceps, we stripped of and partially removed the arachnoid membrane from DS, clivus and 


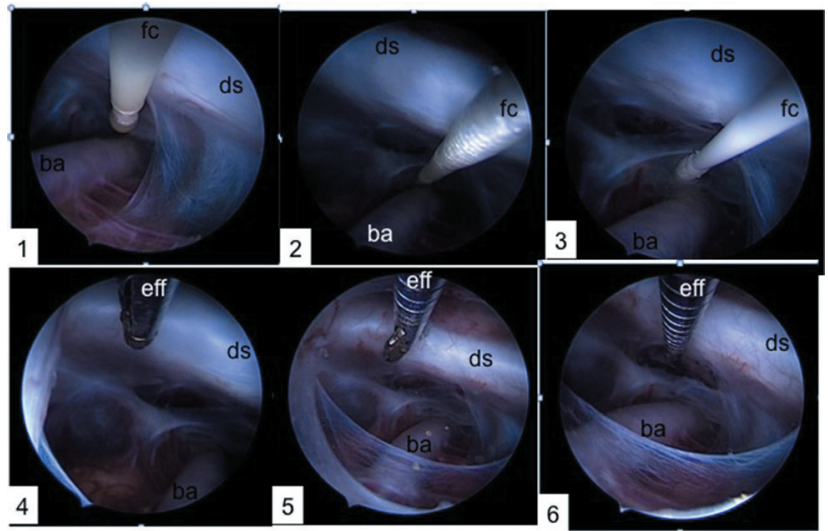

Fig. 7 Peroperative sequential pictures $(1,2,3,4,5$, and 6$)$ of second endoscopic operation of making fenestration in reunited arachnoid and partial removal of arachnoid membrane between BA and DS with FC and EFF. BA, basilar artery; DS, dorsum sella; EFF, endoscopic fenestration forceps; FC, Fogarty catheter.

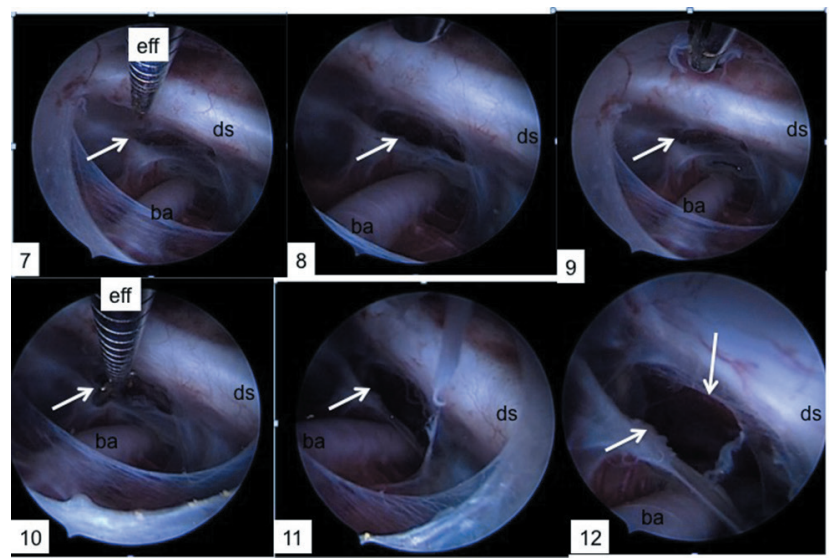

Fig. 8 Continuation of ( - Fig. 7); Peroperative sequential pictures (7, 8, 9, 10,11 , and 12) of second endoscopic operation of making fenestration in reunited arachnoid and partial removal of arachnoid membrane between $\mathrm{BA}$ and DS with FC and EFF, marked by arrows. A part of arachnoid over DS was also removed. BA, basilar artery; DS, dorsum sella; EFF, endoscopic fenestration forceps; FC, Fogarty catheter.

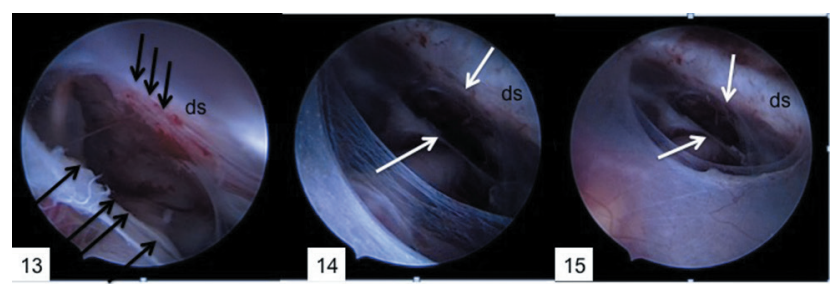

Fig. 9 Continuation of (-Fig. 8); Peroperative sequential pictures $(13,14$, and 15) of second endoscopic operation of making fenestration in reunited arachnoid and partial removal of arachnoid membrane between BA and DS with FC and EFF, marked by arrows. A part of arachnoid over DS was also removed. BA, basilar artery; DS, dorsum sella; $E F F$, endoscopic fenestration forceps; FC, Fogarty catheter.

arachnoid membrane anterior to the $\mathrm{BA}$, taking great care not to injure the vessels and neurostructures, including the third and sixth cranial nerves (-Fig. 7-9). After making a big hole in the reunited membrane, endoscope was removed and the wound was closed accordingly.

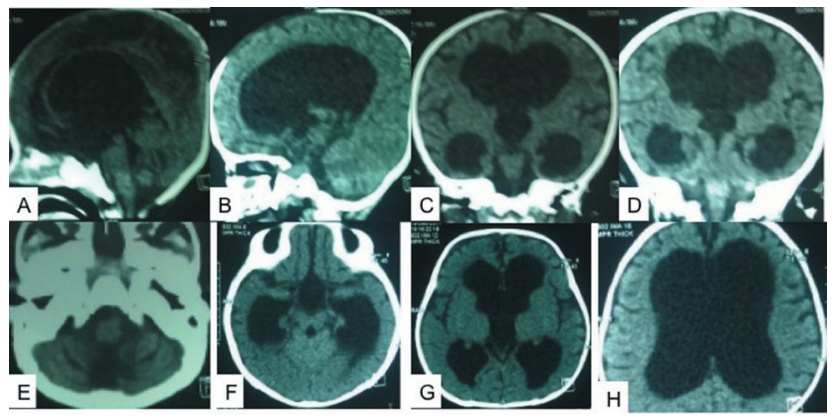

Fig. $10 \mathrm{CT}$ scan of head on 5th postoperative day after second endoscopic operation. (A, B) sagittal, (C, D) coronal, and (E-H) axial images showing reduced size of ventricles with no periventricular edema as well as opening of subarachnoid spaces (note-fourth ventricle became normal in size).

Postoperatively, the patient's respiration became stable with respiration and breast feeding was restarted. His fontanel became lax. CT scan of head on the fourth postoperative day showed decrease size of ventricles, including fourth ventricle with CSF in subarachnoid spaces, and there was marked reduced periventricular hypodensity (-Fig. 10). Then, the patient was symptom-free with normal milestone of development till last follow-up (i.e., 14 months after operation).

\section{Discussion}

FVOO is a very rare cause of obstructive HCP. FVOO usually occurs in pediatric age group and may be congenital, ${ }^{10-12}$ but can occur in adults also. There is no sex differentiation.

Mohanty et $\mathrm{al}^{2}$ reported a case series of 22 patients with FVOO; of these, 10 patients had a medical history, three had suffered intraventricular hemorrhage, and seven patients had infections, including tubercular meningitis, bacterial infection, or prolonged and unexplained fever. The rest of the 12 cases were idiopathic FVOO. Head injury can be a cause of membranous obstruction of CSF and can cause FVOO. ${ }^{3}$

\section{Diagnostic Modalities for FVOO}

The pattern of ventricular enlargement in FVOO is named panventriculomegaly ${ }^{2}$ or tetraventricular HCP. ${ }^{12,13}$ Dilatation or large CSF collection of the foramina of Magendie and Luschka is a characteristic radiological finding in cases of FVOO. ${ }^{4,5,12,14}$ However, it is very difficult to confirm the presence of a membranous obstruction at ventricular outflow via conventional magnetic resonance imaging (MRI). ${ }^{15}$ High-resolution, three-dimensional constructive interference with steady state sequence on $3 \mathrm{~T}$ MRI may be able to detect obstructive membranes, ${ }^{15}$ but this may not be possible in all cases. ${ }^{9}$

The most sensitive diagnostic method is computed tomography (CT) ventriculography, with the injection of contrast medium through a ventricular catheter. ${ }^{11,14}$ Serial CT images after injection will show collected contrast medium in the outlets of the fourth ventricle and subsequent blockage of its diffusion to the prepontine cistern. 
The use of MRI instead of CT as the diagnostic modality for FVOO is recommended to avoid exposure to radiation. ${ }^{16}$

Phase-contrast MRI, ${ }^{5,7}$ cine MRI, 3,4,6,7,10,13,17 or radioisotope cisternogram ${ }^{3,7}$ can be used to see the CSF dynamics.

Direct endoscopic inspection of the fourth ventricle, in case the aqueduct is sufficiently expanded, is another diagnostic option. ${ }^{2}$ Although this technique needs to be done under general anesthesia and carries a risk of damaging the midbrain around the aqueduct, it has been recently reported to be relatively safe. ${ }^{2,13,18,19}$ When MRI is suggestive of FVOO, this technique can allow simultaneous diagnosis and treatment.

Mohanty et $\mathrm{a}^{2}$ reported the entire success rate of ETV for FVOO is $65 \%$ ( 13 successes in 20 cases). Although they did not evaluate the success rates of primary and secondary FVOOs separately, they speculated that failure was attributable to CSF malabsorption as a result of prior meningitis or intraventricular hemorrhage.

Oertel et $\mathrm{al}^{9}$ reported the results of ETV in 20 cases of far distal obstructive HCP where most of the cases were with Dandy Walker or Arnold Chiari malformations; however, there were four cases considered as secondary FVOO. Two of the four patients (50\%) were successfully treated by ETV, while the remaining two patients required early shunting. The success rate of ETV in primary FVOO is 75 to $100 \%$.3,4,8,8,17,20

Most of the failures of ETV for treating FVOO occur within 6 weeks of surgery and that subsequent endoscopic re-exploration revealed patency at the fenestration site. ${ }^{2}$ In some cases, the recurrent HCP can differ. The recurrence occurs later than 6 weeks and stomal stenosis or obliteration can be the cause, which can be successfully treated with endoscopic re-expansion. Indeed, this suggests that the recurrence of HCP observed in such case is not attributable to CSF malabsorption. In such cases (where recurrent HC does not appear to be caused by malabsorption), repeated ETV would be an effective treatment option. ${ }^{21}$

Direct fenestration of membranous obstruction at the fourth ventricle outlets is another previously reported treatment option. ${ }^{8,18,19}$ However, its usefulness is still unclear because it was used in combination with ETV in most cases.

Regarding ETV in infant, some series showed that failure rate can be very high and can be even higher in neonates., ${ }^{2,719,20,22}$ But at the same time, more recent series showed more success of ETV in infants and neonates. ${ }^{22,23}$

Our case was a unique case of primary FVOO with classical CT findings in neonatal age associated with other congenital conditions such as ileal atresia and laryngomalacia.

During ETV, we preferred it to ventriculo-pleural (V-P) shunt as:

1. HCP was of an obstructive variety.

2. Simple, quick, and less traumatic.

3. It is effective even in neonates. ${ }^{22}$

4. H/O laparotomy and ileostomy in a small abdomen where shunt insertion may be difficult with failure and complication.

5. V-P or ventriculo-atrial shunts may cause more respiratory problem (as patient had laryngomalacia).

\section{Shunt is standby procedure when ETV fails.}

But there were some concerns such as the following:

Result of ETV in FVOO is not well known.

Failure chance of ETV is more in neonates.

No or very minimum space between brain stem and DS and clivus.

Chance of thick Liliquist membrane.

To work in a very narrow deep space, with important surrounding vessels and microvessels

Chances of damage to third and sixth cranial nerves.

During second endoscopic procedure, we preferred it as:

1. To confirm the cause-where we found stoma was alright but arachnoid reunited to form a membrane that obstructed CSF flow.

2. If cause is found that can be treated simultaneously (here, we found the cause and treated it with endoscopy).

3. Shunt is standby procedure.

Before second endoscopy, we reviewed the 1st operation's video, where we noticed a large thick flap/band of arachnoid remained flapping in front of BA and posterior to clivus that reunited with the DS arachnoid membrane and surrounding arachnoids to stop CSF flow (- Figs. 3 and $\mathbf{6}$ ). We thought if we were removing (partially /totally) or coagulating this "culprit" arachnoid flap with removal of the arachnoid on DS, we could prevent recurrence of HCP.

So, during re-endoscopic procedure when we found "the thought culprit arachnoid flap" reunited, we perforated it and removed it partially (-Figs. 7-9). At the same time, we also removed the arachnoids on DS. We think, arachnoid on DS has a very important role in recurrence of HCP after ETV with Liliquist membrane penetration, especially in infants.

\section{Conclusion}

From a single case, it was difficult to make a conclusion but, in idiopathic FVOO, ETV with wide fenestration of Liliequist membrane, preferably with partial removal (or judicial coagulation) along with partial removal of arachnoid on DS, may be very useful in treating HCP and preventing recurrent HCP even in infants.

\section{Funding}

None.

\section{Conflict of Interest}

None declared.

\section{References}

1 Ferrer E, de Notaris M. Third ventriculostomy and fourth ventricle outlets obstruction. World Neurosurg 2013; 79(2, Suppl):20.e9-20.e13

2 Mohanty A, Biswas A, Satish S, Vollmer DG. Efficacy of endoscopic third ventriculostomy in fourth ventricular outlet obstruction. Neurosurgery 2008;63(5):905-913

3 Suehiro T, Inamura T, Natori Y, Sasaki M, Fukui M. Successful neuroendoscopic third ventriculostomy for hydrocephalus and syringomyelia associated with fourth ventricle outlet obstruction. Case report. J Neurosurg 2000;93(2):326-329 
4 Karachi C, Le Guérinel C, Brugières P, Melon E, Decq P. Hydrocephalus due to idiopathic stenosis of the foramina of Magendie and Luschka. Report of three cases. J Neurosurg 2003;98(4):897-902

5 Huang YC, Chang CN, Chuang HL, Scott RM. Membranous obstruction of the fourth ventricle outlet. A case report. Pediatr Neurosurg 2001;35(1):43-47

6 Carpentier A, Brunelle F, Philippon J, Clemenceau S. Obstruction of Magendie's and Luschka's foramina. Cine-MRI, aetiology and pathogenesis. Acta Neurochir (Wien) 2001;143(5):517-521

7 Choi JU, Kim DS, Kim SH. Endoscopic surgery for obstructive hydrocephalus. Yonsei Med J 1999;40(6):600-607

8 Longatti P, Fiorindi A, Martinuzzi A, Feletti A. Primary obstruction of the fourth ventricle outlets: neuroendoscopic approach and anatomic description. Neurosurgery 2009;65(6):1078-1085

9 Oertel JM, Mondorf Y, Schroeder HW, Gaab MR. Endoscopic diagnosis and treatment of far distal obstructive hydrocephalus. Acta Neurochir (Wien) 2010;152(2):229-240

10 Inamura T, Morioka T, Nishio S, Ikezaki K, Nonaka H, Yoshiura T. Diverticular enlargement of the foramina of Luschka and congenital hydrocephalus. Childs Nerv Syst 2002;18(11):652-655

11 Rifkinson-Mann S, Sachdev VP, Huang YP. Congenital fourth ventricular midline outlet obstruction. Report of two cases. J Neurosurg 1987;67(4):595-599

12 Takami H, Shin M, Kuroiwa M, Isoo A, Takahashi K, Saito N. Hydrocephalus associated with cystic dilation of the foramina of Magendie and Luschka. J Neurosurg Pediatr 2010;5(4):415-418

13 Longatti P, Basaldella L, Feletti A, Fiorindi A, Billeci D. Endoscopic navigation of the fourth ventricle. Technical note and preliminary experience. Neurosurg Focus 2005;19(6):E12

14 Roth J, Ben-Sira L, Udayakumaran S, Constantini S. Contrast ventriculo-cisternography: an auxiliary test for suspected fourth ventricular outlet obstruction. Childs Nerv Syst 2012;28(3):453-459

15 Dinçer A, Kohan S, Ozek MM. Is all "communicating” hydrocephalus really communicating? Prospective study on the value of 3D-constructive interference in steady state sequence at 3T. Am J Neuroradiol 2009;30(10):1898-1906

16 Joseph VB, Raghuram L, Korah IP, Chacko AG. MR ventriculography for the study of CSF flow. Am J Neuroradiol 2003;24(3):373-381

17 Hashimoto H, Maeda A, Kumano K, Kimoto T, Fujisawa Y, Akai T. Rapid deterioration of primary fourth ventricular outlet obstruction resulting in syndrome of inappropriate antidiuretic hormone secretion. Pediatr Int 2014;56(4):e30-e32

18 Longatti P, Fiorindi A, Feletti A, Baratto V. Endoscopic opening of the foramen of magendie using transaqueductal navigation for membrane obstruction of the fourth ventricle outlets. Technical note. J Neurosurg 2006;105(6):924-927

19 Torres-Corzo J, Sánchez-Rodríguez J, Cervantes D, et al. Endoscopic transventricular transaqueductal Magendie and Luschka foraminoplasty for hydrocephalus. Neurosurgery 2014;74(4):426-435

20 Mohanty A, Anandh B, Kolluri VR, Praharaj SS. Neuroendoscopic third ventriculostomy in the management of fourth ventricular outlet obstruction. Minim Invasive Neurosurg 1999;42(1):18-21

21 Ishi Y, Asaoka K, Kobayashi H, et al. Idiopathic fourth ventricle outlet obstruction successfully treated by endoscopic third ventriculostomy: a case report. Springerplus 2015;4:565

22 Chowdhury FH, Haque MR, Kawsar KA, Sarker MH, Haque AFMM. Hydrocephalus caused by cerebral aqueductal stenosis in infant patients. Indian J Neurosurg 2017;6:189-197

23 Sobti S, Choudhary A, Bhaskar S, Gupta LN. Role of endoscopic third ventriculostomy in shunt malfunction. IJNS 2017;6(2):99-102 